\title{
Fast downflows in a chromospheric filament
}

\author{
K. Sowmya ${ }^{1}$, A. Lagg ${ }^{1}$, S. K. Solanki ${ }^{1,2}$ and J. S. Castellanos Durán ${ }^{1}$ \\ ${ }^{1}$ Max-Planck-Institut für Sonnensystemforschung \\ Justus-von-Liebig-Weg 3, 37077 Göttingen, Germany \\ email: krishnamurthy@mps.mpg.de \\ ${ }^{2}$ School of Space Research, Kyung Hee University, YongIn, Gyeonggi 446-701, Korea
}

\begin{abstract}
An active region filament in the upper chromosphere is studied using spectropolarimetric data in He I $10830 \AA$ from the GREGOR telescope. A Milne-Eddingon based inversion of the Unno-Rachkovsky equations is used to retrieve the velocity and the magnetic field vector of the region. The plasma velocity reaches supersonic values closer to the feet of the filament barbs and coexist with a slow velocity component. Such supersonic velocities result from the acceleration of the plasma as it drains from the filament spine through the barbs. The line-ofsight magnetic fields have strengths below $200 \mathrm{G}$ in the filament spine and in the filament barbs where fast downflows are located, their strengths range between 100 - $700 \mathrm{G}$.
\end{abstract}

Keywords. Sun: filaments, Sun: chromosphere, Sun: magnetic fields

\section{Introduction}

Solar filaments are dense threads suspended in the chromosphere and held in place by strong magnetic fields in the solar atmosphere. A filament appears dark when observed on the disk with the chromospheric lines such as the He I $10830 \AA$ triplet, as the gas inside it is cooler than the photospheric plasma below. Its structure resembles that of a centipede with the elongated body called 'spine' and the many legs called 'barbs' which are rooted in the solar surface.

High speed downflows have been observed in the filament barbs (see e.g., Joshi et al. 2013 Sasso et al. 2011, 2014) where the magnetic field is more vertical than in the filament itself. Recently, Díaz Baso et al. (2019a b) explored the dynamic and magnetic properties of an active region (AR) filament observed with the ground based $1.5 \mathrm{~m}$ GREGOR telescope (Schmidt et al. 2012), with an emphasis on understanding its magnetic topology. An overview about chromospheric filaments can be found in these papers and the references therein. We study the same AR filament that was studied by these authors but focus on the line-of-sight (LOS) velocity distribution and magnetic field strength at selected locations in the barbs.

\section{Observations}

The spectropolarimetric raster scan of the filament associated with the AR 12087 was recorded on 17 June 2014 using the GREGOR Infrared Spectrograph (GRIS; Collados et al. 2012). The scan (ID: 17jun14.005) lasted approximately 15 minutes (09:13 UT - 09:27 UT), covering about $19^{\prime \prime}$ in the scan direction (x-axis) and $63^{\prime \prime}$ along the slit (y-axis), with a step size of $0.126^{\prime \prime}$ and a pixel size of $0.135^{\prime \prime}$ along the slit. The coordinates of the center of the field-of-view (FOV) correspond to a heliocentric angle of $23^{\circ}(\mu=\cos \theta=0.92$, $x=+180^{\prime \prime}$ and $\left.y=-320^{\prime \prime}\right)$. The data was reduced with the standard GRIS data reduction software. The image resolution determined by averaging the power spectrum along the slit direction is $0.5^{\prime \prime}$. The observed spectral window of about $18 \AA$ includes the 

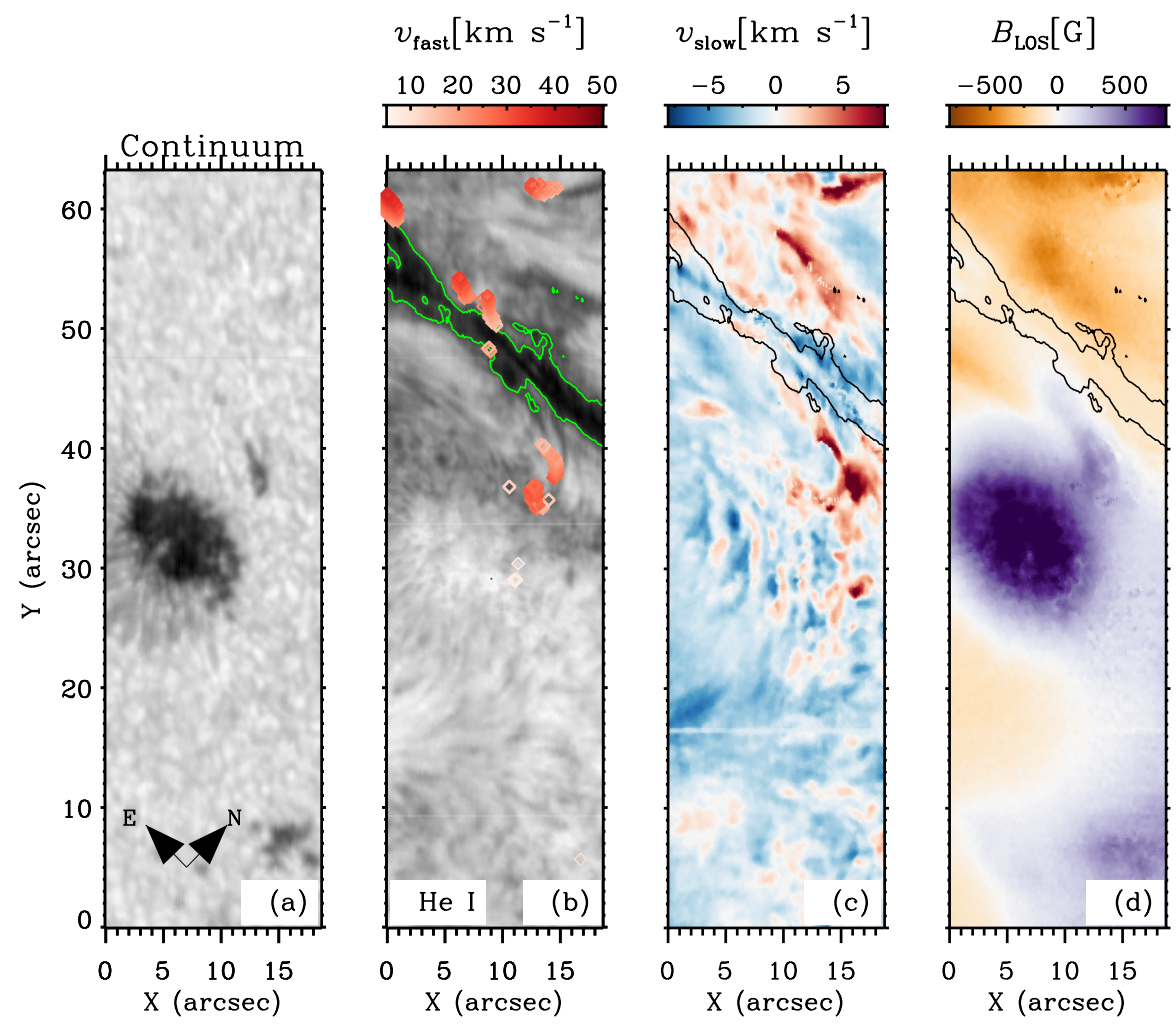

Figure 1. Panel (a): continuum intensity image with the arrows showing the directions of solar north and east. Panel (b): normalized intensity in the He I line at $10830.34 \AA$ with the overplotted colored symbols representing the LOS velocity of the fast component. Panel (c): LOS velocity map for the slow component. Panel (d): LOS magnetic field strength map for the slow component saturated at $\pm 800 \mathrm{G}$. The green and black contours at $0.6 I_{c}$ mark the filament spine.

chromospheric He I $10830 \AA$ triplet, photospheric Si I $10827 \AA$ and Ca I $10839 \AA$ lines and a telluric blend at $10832 \AA$. The average noise in the Stokes parameters is $1.2 \times 10^{-3} I_{c}$, where $I_{c}$ is the continuum intensity. Panels (a) and (b) in Fig. 11 show the intensity images in the continuum redward of the Si line and at He I 10830.34 A. In the observed FOV, a part of the filament spine and a few barbs are visible in He I.

\section{Method}

We invert the spectropolarimetric data using the HeLIx ${ }^{+}$inversion code assuming Milne-Eddington atmosphere and taking into account the Zeeman effect in the incomplete Paschen-Back regime (Lagg et al. 2004, 2009). The Stokes profiles at some locations along the filament barbs show the presence of a second velocity component which is strongly redshifted. A single component inversion does not yield a good fit to such profiles (see panels (a) and (b) in Fig. 2). Therefore, we invert the He I triplet by using two atmospheric components which are mixed through a filling factor. We call the two components 'slow' $\left( \pm 10 \mathrm{~km} \mathrm{~s}^{-1}\right)$ and 'fast' (downflows up to $\left.50 \mathrm{~km} \mathrm{~s}^{-1}\right)$. From such inver- 
sions, we retrieve the LOS velocity and the magnetic field vector for the two atmospheric components.

\section{Results}

The maps of intensity, LOS velocity and LOS magnetic field strength for the slow component are shown in Fig. 1. The green and black contours at $0.6 I_{c}$ identify the filament spine. The slow component is nearly at rest and its LOS velocity (see panel (c)) follows a Maxwellian distribution. The LOS magnetic field strength map (see panel (d)) is saturated at $\pm 800 \mathrm{G}$. Chrompsheric magnetic fields stronger than $800 \mathrm{G}$ are clearly present in the sunspot. The filament is located above the polarity inversion line where the magnetic field is changing sign. Inside the filament spine, the LOS component of the magnetic field is weaker than $200 \mathrm{G}$, in agreement with the previous reports (see e.g., Kuckein et al. 2009).

The locations where a fast component with a filling factor greater than $20 \%$ and a signal in any Stokes polarization parameter higher than three times the noise (within $\pm 0.35 \AA$ from it's wavelength position) is present are marked by red symbols in the He I intensity image in Fig. 1. The colorbar indicates the velocity of the fast component. We found no location where more than two atmospheric components are present. It is apparent that the fast component lies along the filament barbs. The velocity seems to be increasing down the barb reaching supersonic values (higher than the local sound speed of $\sim 10 \mathrm{~km} \mathrm{~s}^{-1}$ ) closer to the footpoints. This increase can be explained by the gravitational acceleration of the plasma from the spine as it drains along the barbs.
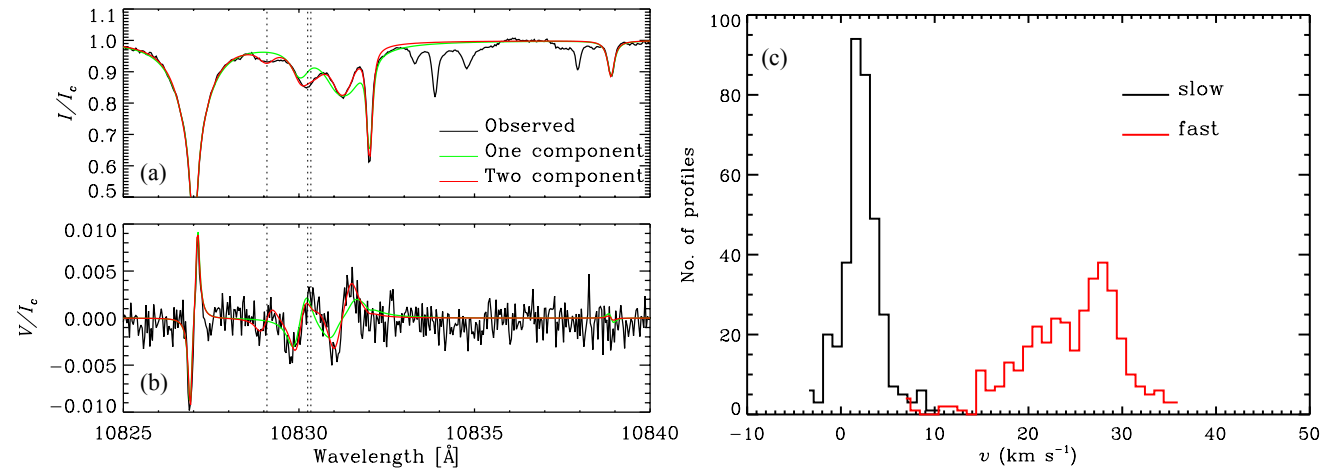

Figure 2. Panel (a): observed intensity profile (black) at a location with the coordinates $x=8.7^{\prime \prime}$ and $y=52.5^{\prime \prime}$ (see Fig. 1), a fit to the observed profile obtained from one component inversion (green) and a fit from two component inversion (red). Panel (b): same as panel (a) but for Stokes $V$. Panel (c): histograms of the LOS velocity at locations where both the slow (black) and the fast (red) components are present.

Panels (a) and (b) in Fig. 2 show Stokes $I$ and $V$ profiles at one of the locations corresponding to $x=8.7^{\prime \prime}$ and $y=52.5^{\prime \prime}$, where the fast component has a velocity of $\sim 30 \mathrm{~km} \mathrm{~s}^{-1}$. Stokes $Q$ and $U$ signals are close to the noise level and hence we do not show them here. The observed profiles are shown in black, the fits from one component and two component inversions are shown in green and red, respectively. Clearly, two components are needed to properly model such anomalous profiles. We identified 360 locations where a fast component is present simultaneously with the slow component. Fig. 2(c) shows the 
velocity distribution for the fast (red) and slow (black) components at those locations. Most of the downflow velocities range between $15-30 \mathrm{~km} \mathrm{~s}^{-1}$ and in some extreme cases they reach $35 \mathrm{~km} \mathrm{~s}^{-1}$ or more. Along the filament barbs, the fast downflow component has LOS magnetic field strength ranging between 100 - $700 \mathrm{G}$.

\section{Conclusions}

We studied an active region filament observed by the ground based GREGOR telescope. Using two component Milne-Eddington inversions, we determined the velocity and the magnetic field vector at chromospheric heights where He I forms. We identified supersonic downflows, as high as $35 \mathrm{~km} \mathrm{~s}^{-1}$, coexisting with a slow flow component. The high donwlfow velocities can be understood with the acceleration of the plasma as it drains along the filament barbs. The slow component could be originating from He I layer at a different height or could be due to straylight. We find that the LOS component of the magnetic field is weaker than $200 \mathrm{G}$ within the filament spine. In filament barbs where strong downflows are found, the LOS magnetic field is as strong as $700 \mathrm{G}$.

\section{Acknowledgements}

Marie Skłodowska-Curie grant agreement No. 797715. The 1.5-meter GREGOR solar telescope was built by a German consortium under the leadership of the Leibniz Institut für Sonnenphysik in Freiburg with the Leibniz Institut für Astrophysik Potsdam, the Institut für Astrophysik Göttingen, and the Max-Planck Institut für Sonnensystemforschung in Göttingen as partners, and with contributions by the Instituto de Astrofísica de Canarias and the Astronomical Institute of the Academy of Sciences of the Czech Republic. The GRIS instrument was developed thanks to the support by the Spanish Ministry of Economy and Competitiveness through the project AYA2010-18029 (Solar Magnetism and Astrophysical Spectropolarimetry). This study has made use of SAO/NASA Astrophysics Data System's bibliographic service

\section{References}

Collados, M., López, R., Páez, E., Hernández, E., Reyes, M., et al. 2012, AN, 333, 872

Díaz Baso, C. J., Martínez González, M. J., \& Asensio Ramos, A. 2019a, A $\mathscr{J} A$, 625, A128

Díaz Baso, C. J., Martínez González, M. J., \& Asensio Ramos, A. 2019b, A 6 A, 625, A129

Joshi, A. D., Srivastava, N., Mathew, S. K., \& Martin, S. F. 2013, SoPh, 288, 191

Kuckein, C., Centeno, R., Martínez Pillet, V., Casini, R., Manso Sainz, R., \& Shimizu, T. 2009, $A \& A, 501,1113$

Lagg, A., Woch, J., Krupp, N., \& Solanki, S. K. 2004, A\& A, 414, 1109

Lagg, A., Ishikawa, R., Merenda, L., Wiegelmann, T., Tsuneta, S., \& Solanki, S. K. 2009, ASPC, 415,327

Sasso, C., Lagg, A., \& Solanki S. K. 2011, A\& A, 526, A42

Sasso, C., Lagg, A., \& Solanki S. K. 2014, A\& A, 561, A98

Schmidt, W., von der Lühe, O., Volkmer, R., Denker, C., Solanki, S. K., et al. 2012, Astronomische Nachrichten, 333, 796 